This PDF is a selection from a published volume from the National Bureau of Economic Research

Volume Title: Frontiers in Health Policy Research, Volume 7

Volume Author/Editor: David M. Cutler and Alan M. Garber, editors

Volume Publisher: MIT Press

Volume ISBN: 0-262-03325-9

Volume URL: http://www.nber.org/books/cutl04-1

Conference Date: June 6, 2003

Publication Date: July 2004

Title: Disability Forecasts and Future Medicare Costs

Author: Jayanta Bhattacharya, David M. Cutler, Dana P. Goldman, Michael D. Hurd, Geoffrey F. Joyce, Darius N. Lakdawalla, Constantijn W. A. Panis, Baoping Shang

URL: http://www.nber.org/chapters/c9870 


\title{
Disability Forecasts and Future Medicare Costs
}

\author{
Jayanta Bhattacharya, Stanford University and NBER \\ David M. Cutler, Harvard University and NBER \\ Dana P. Goldman, RAND and NBER \\ Michael D. Hurd, RAND and NBER \\ Geoffrey F. Joyce, RAND \\ Darius N. Lakdawalla, RAND and NBER \\ Constantijn W. A. Panis, RAND \\ Baoping Shang, RAND
}

\section{Executive Summary}

The traditional focus of disability research has been on the elderly, with good reason. Chronic disability is much more prevalent among the elderly, and it has a more direct impact on the demand for medical care. It is also important to understand trends in disability among the young, however, particularly if these trends diverge from those among the elderly. These trends could have serious implications for future health care spending because more disability at younger ages almost certainly translates into more disability among tomorrow's elderly, and disability is a key predictor of health care spending.

Using data from the Medicare Current Beneficiary Survey (MCBS) and the National Health Interview Study (NHIS), we forecast that per-capita Medicare costs will decline for the next fifteen to twenty years, in accordance with recent projections of declining disability among the elderly. By 2020, however, the trend reverses. Per-capita costs begin to rise due to growth in disability among the younger elderly. Total costs may well remain relatively flat until 2010 and then begin to rise because per-capita costs will cease to decline rapidly enough to offset the influx of new elderly people. Overall, cost forecasts for the elderly that incorporate information about disability among today's younger generations yield more pessimistic scenarios than those based solely on elderly data sets, and this information should be incorporated into official Medicare forecasts.

\section{Introduction}

To help the government take the actions necessary to keep Medicare solvent, the Center for Medicare and Medicaid Services (CMS) needs to generate accurate predictions for future health care spending. This requires predicting how many people of various types will be alive in each future year and what their health care spending will be. 
The first part of this exercise is to project population size. Official projections of the aged beneficiary population by age and gender are available from prominent sources such as the Social Security Administration (SSA). These projections already account for the longrun trends in decreasing age-specific mortality rates. The SSA population estimates make it clear that the baby boomers will swell the ranks of the elderly significantly starting in 2010. Forecasting per-capita health expenditures for people of a given age is a more difficult proposition. Individual health care spending is influenced by many factors: age, gender, health status, diseases and the medical technology to treat them, the price of care, insurance coverage, living arrangements, and care from family and friends. Spending estimates are uncertain because it is difficult to predict changes in these factors and in their relationships to health spending. One can assume, as most actuarial models do, that health care spending remains constant within a given age-gender category. In that case, estimated future Medicare expenditures are influenced only by changes in the age composition of the population and general trends in spending that are applied uniformly across agegender categories. This approach overlooks several key factors, however, including the importance of changes in disability and health status among the future elderly.

Disability has been shown in numerous contexts to be an important predictor of elderly health care costs. For example, Goldman et al. (2003) find that elderly with multiple activity limitations can have up to ten times higher mean Medicare costs than those with no limitations, and similar patterns occur in median spending. One possibility is that disability simply reflects a different disease, but even within chronic diseases, they find that elderly with greater disability can have up to three times higher spending. Clearly Medicare forecasts will be highly sensitive to disability trends.

In this paper, we present a natural and straightforward method for forecasting elderly disability from observed disability among the young. The principal forecasting problem, is that trends in disability among the young have not been stable or consistent. Overall, disability among the young has been increasing somewhat, but the rate of increase has varied substantially. For example, the early 1990s saw the sharpest and most dramatic increases in disability among the young, while the late 1980s and mid 1990s saw more muted growth. These different rates of increase have completely different implications for future health care expenditures. 


\section{Methods}

\section{Data}

We use information from the National Health Interview Survey (NHIS) and the Medicare Current Beneficiary Survey (MCBS) to predict disability and the MCBS to predict costs. The NHIS is a nationally representative set of individual-level data on demographics and health status and is designed to represent the noninstitutionalized population. It has been collected every year since 1957. The stability of the NHIS survey design makes it particularly attractive for analyzing longrun trends in disability. Although the survey was redesigned in 1982 and 1997, it is possible to construct consistent estimates from 1984 to 1996. The MCBS is a nationally representative sample of the Medicare population. Because nearly all permanent U.S. residents over age 65 are eligible for Medicare, we can use this population segment as a sample of the over- 65 population. The MCBS has the advantage of including the institutionalized population as well as the noninstitutionalized. We use the NHIS to measure disability for people under age 65 but the MCBS to measure disability for people over age 65 . Below, we describe a procedure for producing a complete age-profile of disability by reconciling the disability estimates across the two data sets.

The MCBS is a nationally representative data set designed to ascertain utilization and expenditures for the Medicare population, especially those expenditures borne by the beneficiary or supplemental insurance. The sample frame consists of aged and disabled beneficiaries enrolled in Medicare Part A and/or Part B, although we use only the aged. The MCBS attempts to interview each person twelve times over three years, regardless of whether he or she resides in the community, resides in a facility, or moves between community and facility settings. The disabled (under 65 years of age) and the oldest old (85 years of age or over) are oversampled. The first round of interviewing was conducted in 1991. Originally, the survey was a longitudinal sample with periodic supplements and indefinite periods of participation. In 1996, the MCBS switched to a rotating panel design with limited periods of participation. Each fall, a new panel is introduced, with a target sample size of 12,000 respondents, and each summer a panel is retired. The MCBS contains detailed self-reported information, including the prevalence of various conditions, measures of physical limitation in performing activities of daily living (ADLs) and instrumental activities of daily living (IADLs), and height and weight. In addition, 
the MCBS contains detailed self-reported data on health service use, as well as Medicare service use records. Institutionalized respondents are interviewed by proxy. To measure disability, we use the ADL questions in the MCBS. Specifically, respondents are asked whether they have any difficulty with bathing or showering, dressing, eating, getting in or out of chairs, walking, and using the toilet. Therefore, an individual in the MCBS can have anywhere from zero to six ADLs.

The NHIS can be used to measure disability among those under age 65 according to a similar measure, but the ADL questions are not asked in the NHIS until 1995. This feature is troublesome because we wish to project trends in future health in part by examining long-run trends in past disability. The NHIS does ask earlier questions about disability from 1982 onward. In the 1995 NHIS, respondents are asked both the ADL questions and the earlier disability questions. This feature will allow us to link the earlier disability measures with the ADL measures and thus construct measures of ADL limitations going back to 1982.

From 1982 to 1995, the NHIS began asking all respondents over age 60 , as well as all those age 5 to 59 who reported some activity limitation, if they needed help with personal care. ${ }^{1}$ Based on a respondent's answer to the personal care question, she or he was placed in one of three categories: (1) unable to tend to personal care needs, (2) limited in performing other routine needs, (3) not limited in personal care or routine needs. In a 1995 disability supplement, the NHIS asked both these personal care questions as well as another set more similar to the MCBS questions. The NHIS reports whether individuals have any difficulty with bathing or showering, dressing, eating, getting in and out of bed or chairs, walking, or using the toilet. These questions are more similar to the MCBS, but they were asked only in 1995. To construct estimates for earlier years, we use the 1995 data to construct a map from the personal care variables to the number of ADLs. This is done using an ordered probit regression where the dependent variable is the number of ADLs, and the independent variables are dummy variables for an individual's personal care needs, age, gender, and race.

The result of this procedure is a single, combined data set representative of the entire population age 25 and over. ${ }^{2}$ Each person in the sample is assigned several ADLs, along with other reported demographic characteristics. We characterize the disability of every individual in the sample by placing her or him in one of three mutually exclusive disability states: institutionalized, having zero ADL limitations, or having at least one ADL. All individuals in the latter two 
categories are noninstitutionalized. Defining nursing home residence as a disability state allows us to use the 1997 and 1998 years of the MCBS, during which disability among the institutionalized is measured differently. Because the NHIS represents our only data source for people under age 65 , and because it is confined to the noninstitutionalized, we are forced to assume a zero rate of institutionalization for people under 65 .

The rates of disability by age group are shown in table 3.1. A sharp break in reported disability occurs at age 65 , when we switch from the NHIS data to the MCBS data. In general, reported rates of disability are much lower in the NHIS than those in the MCBS. Some of this difference can be attributed to different wording in survey questions, but rates are lower in the NHIS even for identically worded questions. This issue is discussed in more detail in Goldman et al. (2003). We propose a method for dealing with this discrepancy in the following section.

\section{Forecasting Disability}

To forecast disability, we first construct smooth age-prevalence profiles of disability from the 1992-1996 data sets. The smoothing procedure is

Table 3.1

Prevalence of disability by age group ${ }^{a}$

\begin{tabular}{llll}
\hline Age group & Zero ADLs & Some ADLs & Nursing home \\
\hline $25-29$ & 99.72 & 0.28 & - \\
$30-34$ & 99.56 & 0.44 & - \\
$35-39$ & 99.4 & 0.6 & - \\
$40-44$ & 99.29 & 0.71 & - \\
$45-49$ & 99.17 & 0.83 & - \\
$50-54$ & 98.95 & 1.05 & - \\
$55-59$ & 98.6 & 1.4 & - \\
$60-64$ & 97.81 & 2.19 & - \\
$65-69$ & 79.75 & 18.74 & 1.51 \\
$70-74$ & 76.43 & 21.31 & 2.26 \\
$75-79$ & 67.22 & 28.22 & 4.57 \\
$80-84$ & 54.53 & 34.74 & 10.73 \\
$85-89$ & 37.99 & 39.82 & 22.19 \\
$90+$ & 19.66 & 40.67 & 39.66 \\
\hline
\end{tabular}

aFigures for people younger than 65 are based on 1992-1996 NHIS. Figures for people older than 65 are based on 1992-1996 MCBS. Figures for the elderly should not be compared to the figures for people younger than 65 for reasons discussed in the paper. 
described in the appendix. We perform smoothing separately for the population under age 65 in the NHIS and then for the population over age 65 in the MCBS. ${ }^{3}$ The NHIS and MCBS results are based on different questionnaires, so we do not simply combine the two prevalence profiles. Instead, we take the MCBS prevalence profile as the measure of disability for people over age 65 . To construct the profile for the under- 65 population, we combine estimates of disability incidence from the NHIS with the MCBS prevalence for people who are 65. For example, to calculate the prevalence of disability among 64-year-olds, we take the MCBS prevalence among 65-year-olds and then subtract the incident cases of disability for people between the ages of 64 and 65 in the NHIS. In this manner, we extrapolate backward in age, back to age 25. This method yields a full age-prevalence profile that is, broadly speaking, comparable to the levels in the MCBS measurement scheme.

Using these age-prevalence profiles, we calculate the extent to which aging raises the prevalence of disability (in more formal language, we compute age-incidence profiles). This approach allows us to age the 1996 population forward through the life cycle and construct forecasts of disability in the future. Suppose, for example, that the prevalence of disability rose by an average of 2 percentage points between the ages of 65 and 66, and suppose that 65-year-olds in 1996 exhibited a 10 percent prevalence of disability. We would thus forecast that 66 -year-olds in 1997 would exhibit a 12 percent prevalence of disability. This approach allows us to compute the forecasted prevalence of disability at any age and future year $t$. Call this prevalence of disability $d(t, a)$. To forecast the number of disabled people at each age and year, we use population projections. The Census Bureau projects population for single-year age categories for every year until 2100. This yields estimates of $p(t, a)$, the population of people age $a$ in year $t$. The number of disabled people age $a$ in year $t$ is thus $D(t, a)=p(t, a)^{*} d(t, a)$. For simplicity, we treat population growth and disability trends as being independent of each other.

\section{Forecasting Costs}

Given estimates of the disabled population, we need a way to translate disability into health expenditure forecasts. We do this in the context of linear regression. The primary dependent variables used in the cost regressions are Medicare reimbursements and their components (Part A and Part B reimbursements) and total medical expenditures. We use longitudinal data from the Medicare Current Beneficiary 
Survey (MCBS) cost and use files (1992-1998) to estimate average Medicare expenditures for persons age 65 to 90 , conditional on their health status. We regress monthly Medicare reimbursements for each individual in the sample on disability status (i.e., which of the three disability states an individual is in), linear age splines, and health/ age interactions separately for males and females. The coefficients from these models are used to predict monthly Medicare expenditures for each age/gender/health cell. As in the disability forecasts, we use the MCBS respondents over age 65, but for the purposes of estimating cost, we also exclude people enrolled in health maintenance organizations (HMOs) and those without Part B supplemental Medicare insurance, because of incomplete ascertainment of health care utilization. These exclusions yield an average yearly sample of about 9,400 beneficiaries.

Given our cost regressions, we can translate the forecasted size of the disabled population in every year into projected health expenditures. We first compute from the expenditure equation the average health care expenditures for individuals who are age $a$ and of disability status $k$. We call this $e(k, a)$. Assuming the real price of health care is rising with general inflation-an assumption that admittedly understates true price growth (Chernew, Hirth, and Cutler 2003) — total health care expenditures in year are then given by:

$\sum_{a=25}^{\infty} \sum_{k=1}^{3} e(k, a) * D(t, a ; k)$

\section{Results}

\section{Forecasts of Disability}

The resulting forecasts of disability are shown in figures 3.1 through 3.4. The figures show forecasted prevalence of disability for four different age groups. Figure 3.1 tells the story: while the rate of institutionalization does not change, the prevalence of disability among those in the community is predicted to fall until 2015 but then to stabilize and even rise slightly thereafter. The figures make clear that the reversal of disability trends comes from the young cohorts entering old age-in figure 3.2, disability does not decline at any time for individuals age 65 to 74. Disability stops falling in figure 3.3 for 75 - to 84 -year-olds around 2010, while it does not do so until nearly 2020 for those over age 85 in figure 3.4. Indeed, the growth in disability is not all that substantial for this oldest age group. 


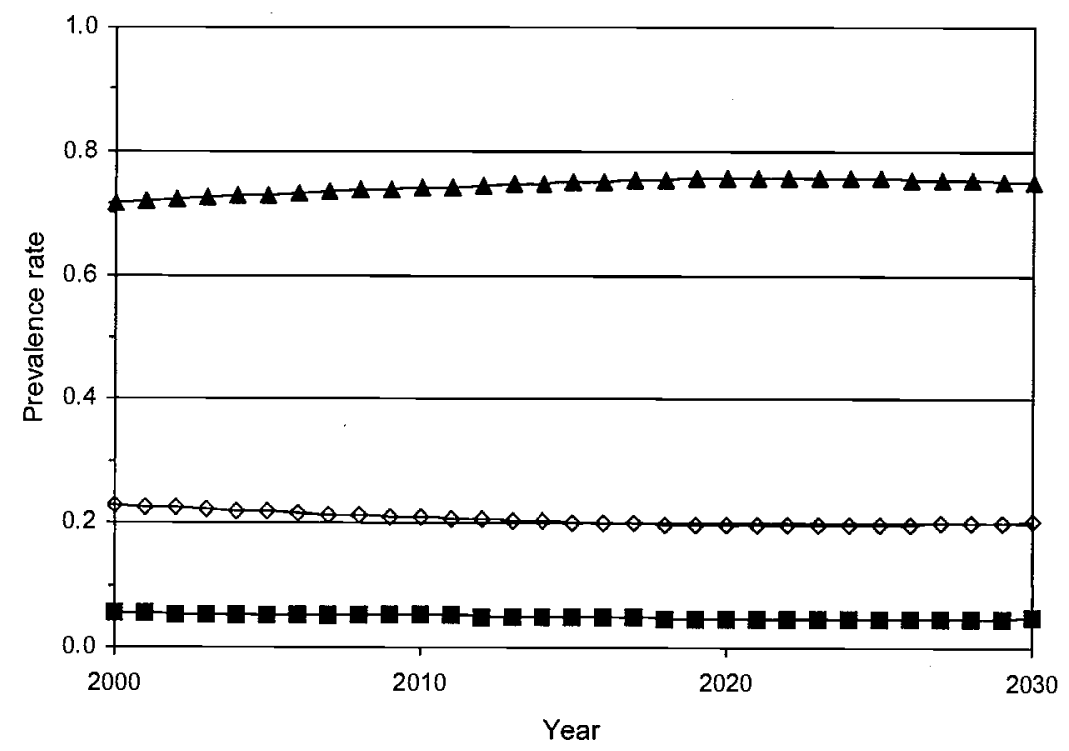

tr No ADLs

- 1 or more ADLs

$\rightarrow$ Nursing home

Figure 3.1

Forecasted prevalence of disability among people age 65 or older

We performed the following check on our disability forecasts. We used NHIS data from the 1980s to forecast disability growth for the population under age 65 from 1990 to $1996 .{ }^{4}$ We then compared these forecasts to actual disability rates in the 1990s. Figure 3.5 displays the results. It is significant that our method understates actual growth in disability over time. The forecast error shifts down as we move from 1990 to 1996. Therefore, our predictions for cost growth should be viewed as perhaps a lower bound on actual cost growth.

\section{Disability and Health Care Costs}

Cost profiles by age and health state are shown in Figures 3.6 and 3.7 for males and females, respectively. Medicare beneficiaries age 65 and older who experience difficulty in walking, dressing, or getting out of bed have substantially higher medical expenditures than those without limitations. Among the noninstitutionalized, for example, persons 

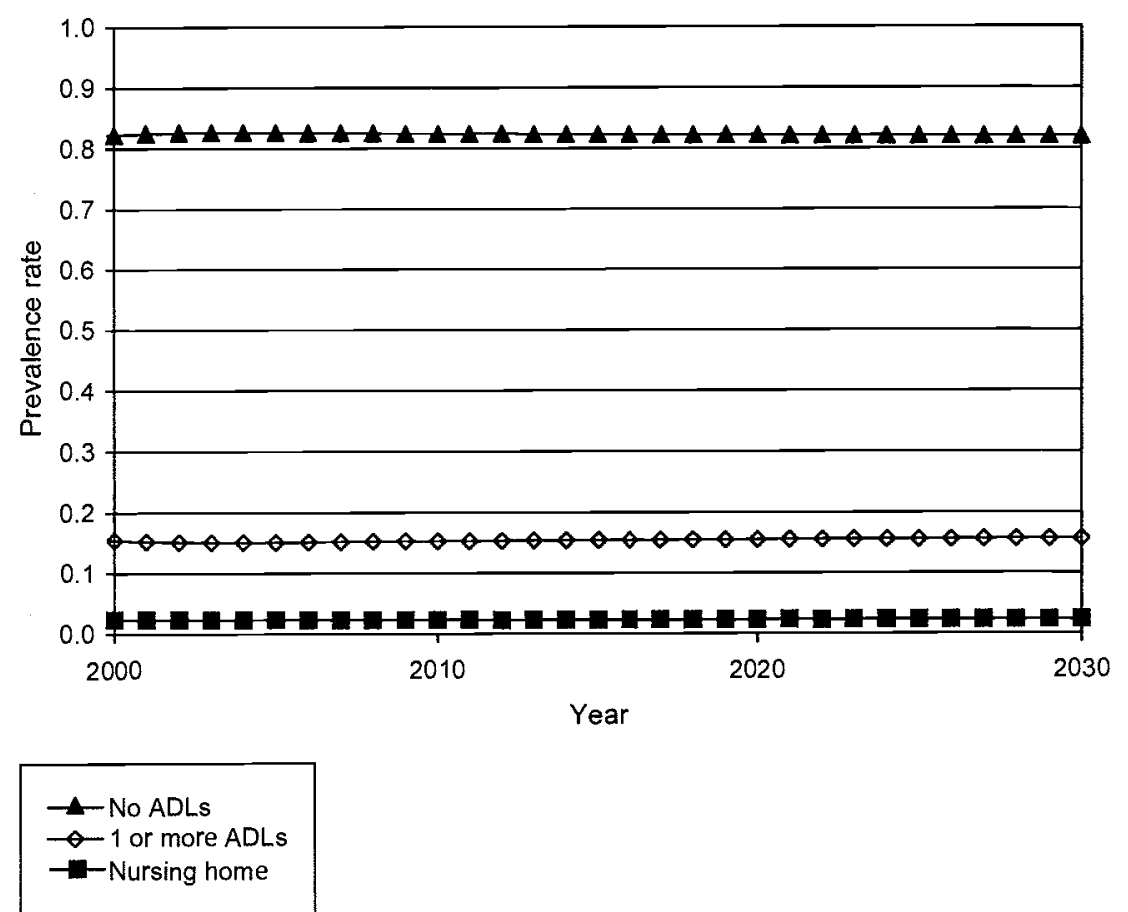

Figure 3.2

Forecasted prevalence of disability among people age 65 to 74

reporting one or more ADLs incur roughly $\$ 500$ more in monthly Medicare expenses than similar-age adults without limitations. Although compositional changes are likely to confound cost profiles, the impact of age on medical expenditures is best observed by examining the costs of healthy beneficiaries (noninstitutionalized, no ADLs). Monthly Medicare costs increase monotonically among the healthy, rising by $\$ 50$ to $\$ 100$ per ten years of life.

The most salient difference across health states is higher monthly expenditures for males. Noninstitutionalized women without ADLs have 25 to 50 percent lower monthly expenditures than similar-age males, and the gap widens in absolute terms as health status worsens. Clear gender differences also exist in the cost profiles of nursing home residents. Average monthly Medicare costs among institutionalized women rise rapidly from age 65 to 70 and then decline consistently for a decade before leveling off. In contrast, average Medicare costs peak 


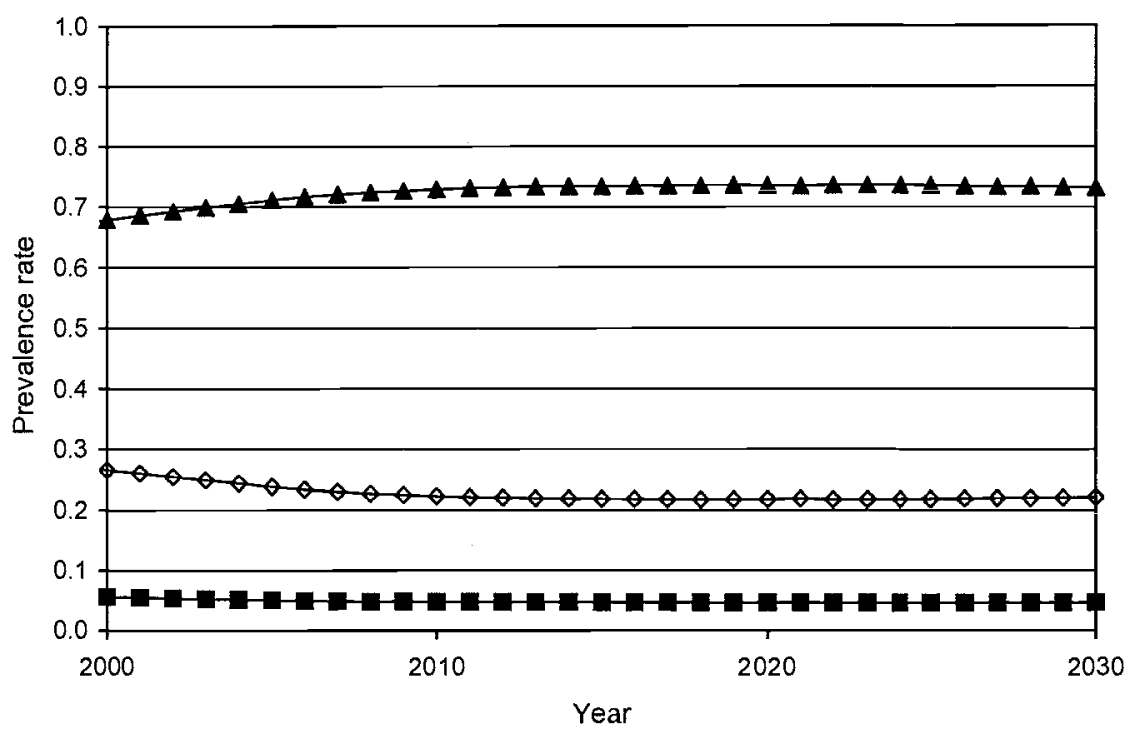

\section{$\rightarrow$ No ADLs \\ $\checkmark 1$ or more ADLs \\ - Nursing home}

Figure 3.3

Forecasted disability rates among people age 75 to 84

around age 75 for institutionalized males and remain near that level thereafter.

\section{Forecasting Health Expenditures}

We combine the age-gender-disability profiles of Medicare costs from the previous section with our forecasts of population in each age-gender-disability cell to produce forecasts of Medicare costs. This results in forecasts of costs that are in terms of blended 1992-1998 dollars; the weights across years are governed by the MCBS sampling scheme. Figure 3.8 displays our forecast of percapita Medicare costs by age group, while figure 3.9 displays forecasted total costs by age group. Figure 3.8 bears out our disability forecasts by showing a slight projected increase in per-capita costs for people age 65 to 74 but declines for those above age 75 . Perhaps more striking is the narrowing of the gap between the per-capita 


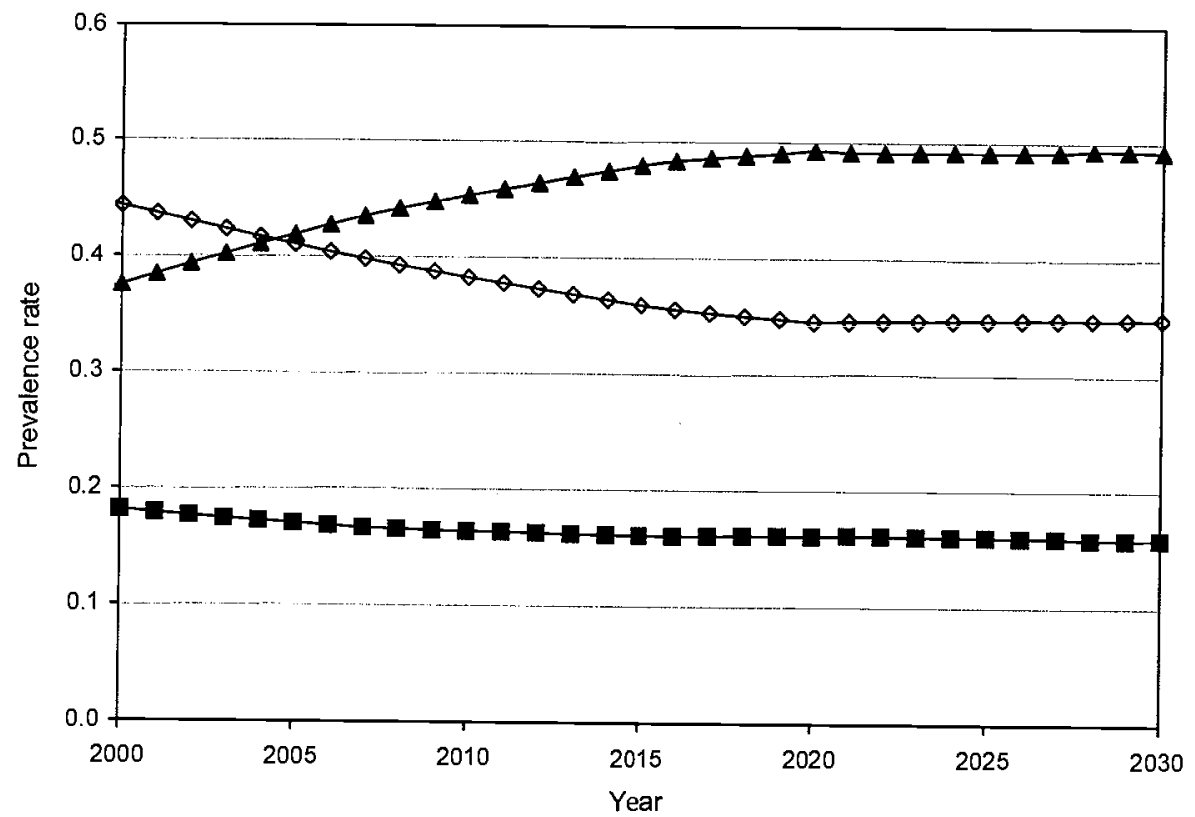

$\simeq$ No ADLs

-1 or more ADLs

- Nursing home

Figure 3.4

Forecasted disability rates among people age 85 or older

costs of 65 to 74-year-olds and the per-capita costs of the entire over65 population. From the start of our forecast period, there is a steady and substantial narrowing of the age gap in per-capita Medicare costs. Total costs look a bit different largely because population growth among the oldest old is projected to be more rapid than among the young old. The important implication of figure 3.9 has less to do with age effects and more to do with timing. Total Medicare costs are projected to be quite flat for the next several years, in spite of population growth, but they begin a steady ascent shortly thereafter. The growth in per-capita costs that we project causes accelerating growth in total costs after 2015. In other words, the future path of Medicare costs is unlikely to resemble its current path mostly because trends in disability may reverse themselves. 

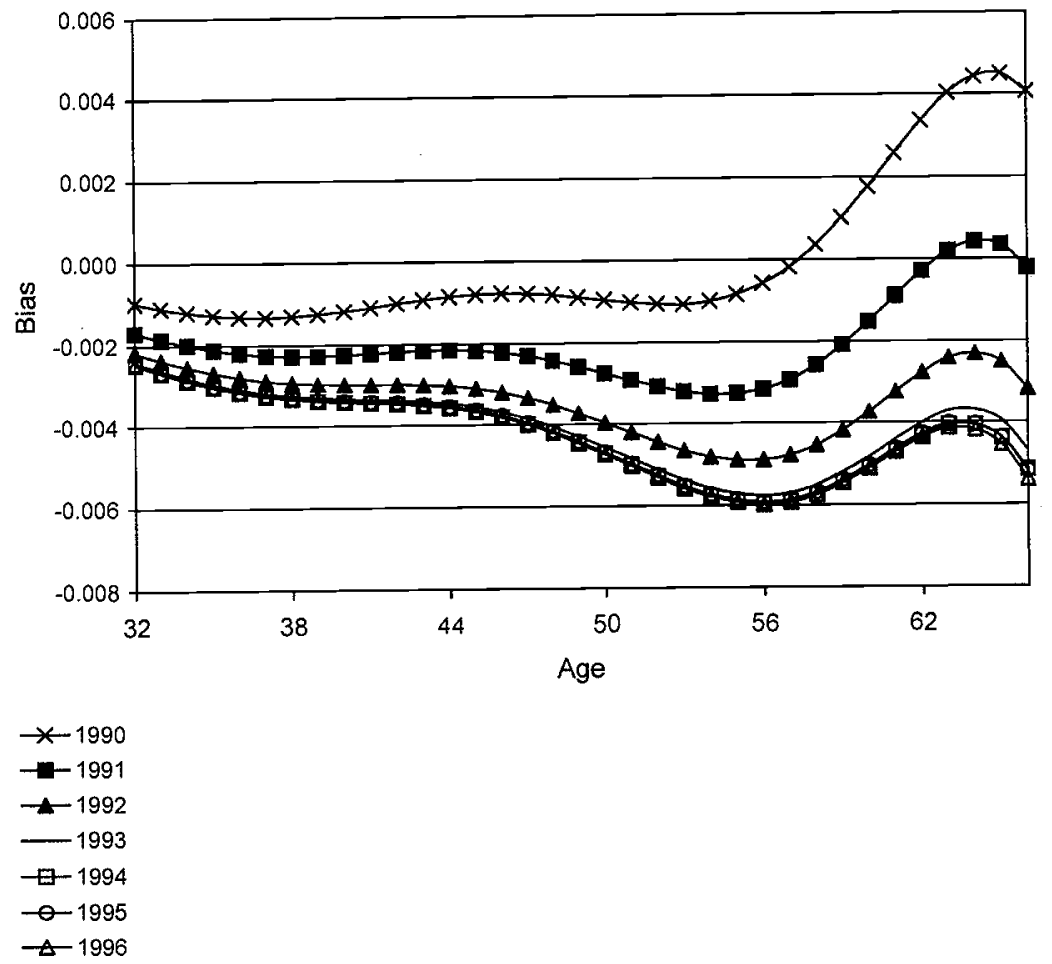

Figure 3.5

Forecast error in NHIS, 1990-1996

The effect of disability on forecasted costs can be seen most clearly if we calculate the implications for costs of different disability scenarios. Figure 3.10 depicts our forecasts for per-capita costs along with the forecasts that would result from three other scenarios: constant disability prevalence, disability declines at the rate experienced from 1989 to 1994 as calculated by Manton et al. (1997) using the National Long Term Care Survey (NLTCS), and disability declines at the rate observed in the NLTCS from 1994 to 1999 (Manton and Gu 2001). Table 3.2 summarizes the differences.

First, it is useful to understand future costs in the context of constant disability. Only the age and gender structure of the population changes under this scenario. Aging is expected to reduce per-capita costs somewhat between 2005 and 2020 with the influx of the young old who will accompany the aging of the baby-boom cohort. By 2020, however, the continued aging of the baby boomers will start to raise per-capita costs. 


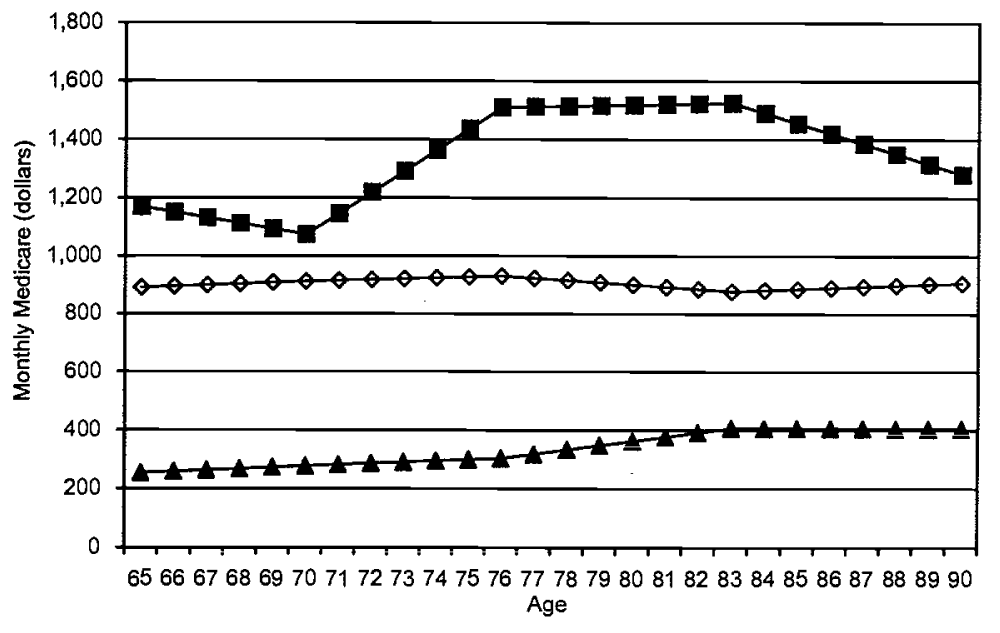

$\rightarrow$ No ADL limitations $\rightarrow 1$ or more ADL limitations $\rightarrow-$ Nursing home

Figure 3.6

Monthly Medicare reimbursement by age and health state for males

Table 3.2

Scenario

Description

Base

Age-prevalence profile changes based on trends in the MCBS (for the elderly population) and the NHIS (for people younger than 65).

Constant

Age-prevalence profile fixed based on initial year. For the entering cohort of 65-year-olds, disability is projected using NHIS prevalence and trend data.

Manton et al (1997)

Age-prevalence profile changes based on trends in NLTCS from 1989-1994 (including the entering cohort of 65-year-olds).

Manton and Gu (2001)

Age-prevalence profile changes based on trends in NLTCS from 1994-1999 (including the entering cohort of 65-year-olds). 


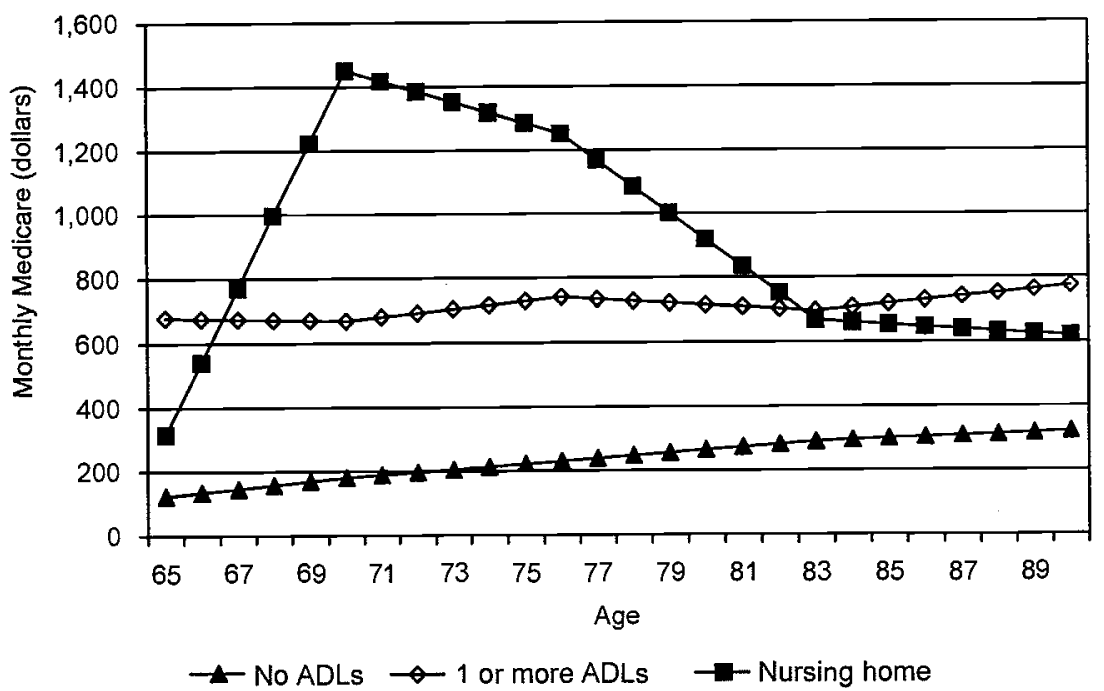

Figure 3.7

Monthly Medicare reimbursement by age and health state for females

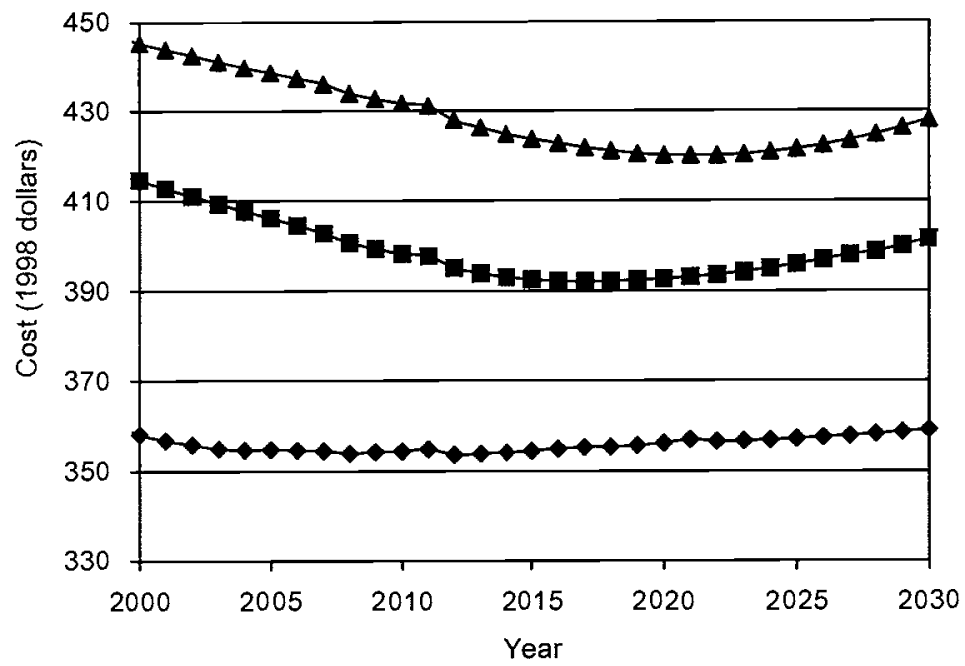

$-65+$

$\rightarrow-65-84$

$\rightarrow-65-74$

Figure 3.8

Forecasts of per-capita monthly Medicare costs by age group 


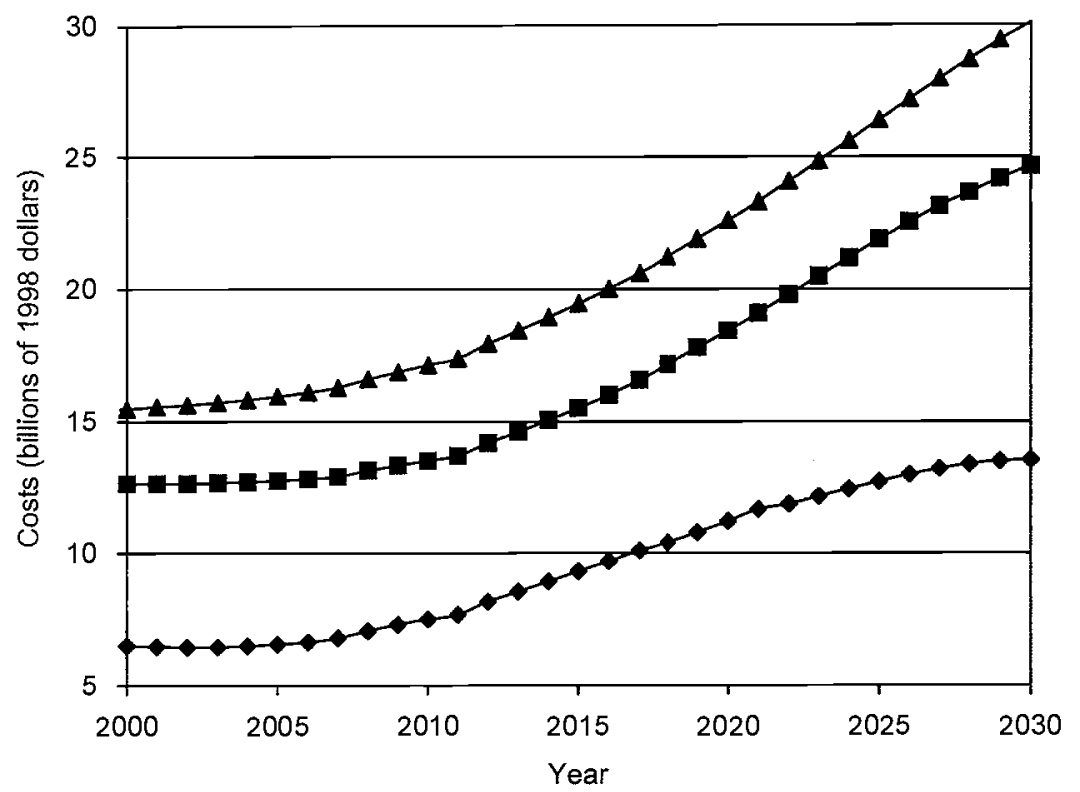

$-165+$

$\rightarrow-65-84$

$\rightarrow-65-74$

Figure 3.9

Forecasts of total monthly Medicare costs by age group

The constant disability curve makes clear, however, that the vast majority of the projected decline in per-capita costs owes itself to future declines in disability. The extent and duration of these declines are then crucial in governing the path of per-capita costs. Three scenarios are possible for disability change shown on the graph: our scenario, one assuming constant rates of decline in disability equal to the decline between 1989 and 1994, and one assuming constant rates in disability equal to the decline between 1994 and 1999. At the start of the forecast period in 2000 , our model actually produces the most optimistic forecasts for per-capita costs. However, the rate of decline from 2000 onward is much slower than in either of the other two scenarios for disability decline. Just after 2010 , per-capita costs in our scenario overtake per-capita costs for the disability decline scenarios. Indeed, by 2018, we forecast that per-capita costs will cease to decline and begin to rise. From 2010 onward, our scenario actually tracks the constant disability 


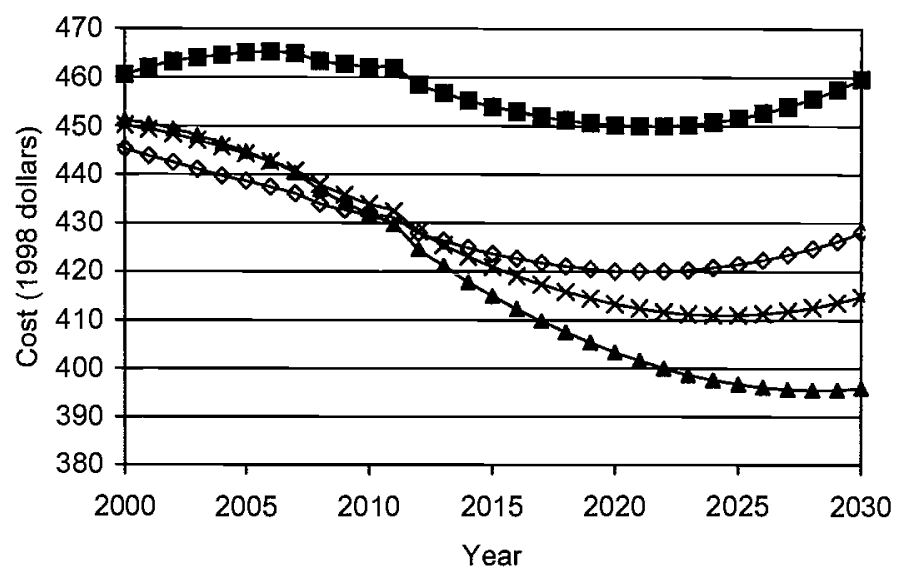

Base

$\rightarrow$-Constant

- Using 1989-1994 (Manton et al. 1997)

$\rightarrow$ Using 1994-1999 (Manton et al. 1997)

Figure $\mathbf{3 . 1 0}$

Impact of disability forecasts on per-capita Medicare costs

Notes: This figure shows projections of per-capita Medicare costs under four scenarios as described in the text. The base case is our projection incorporating information on disability trends from the NHIS (for the young) and the MCBS (for the elderly). The constant case assumes fixed age-prevalence profiles for disability. The other two cases assume changes in the elderly age-prevalence profile based on trends observed in the National Long Term Care Survey and ignore NHIS trends among the young.

case. By 2030, our model predicts real monthly Medicare costs that are $\$ 2$ billion higher (in 1998 dollars) than those implied by the disability declines measured between 1989 and 1994, and $\$ 1$ billion higher than those implied by the declines between 1994 and 1999 .

\section{Conclusions}

Disability is not just a feature of old age. Economic development and technological change in health care have allowed people at all ages to live in frailty with greater ease than at any other time in history. Any analysis of disability must account for changes in disability among the young as well as the elderly. From the mid-1980s to the mid-1990s, the young have reported an increase in disability, even as the old have become relatively healthier. Forecasts based on trends in the disability among the old tend to overstate the benefit of these changes. ${ }^{5}$ 
Our forecasts imply that per-capita Medicare costs will decline for the next fifteen to twenty years; this finding is in accordance with recent declines in disability among the elderly. By 2020, however, percapita costs begin to rise as a result of growth in disability among the young old. As these young-old cohorts age, per-capita costs will continue to grow. Total costs may well remain relatively flat until 2010 and then begin to rise as per-capita costs will cease to decline rapidly enough to offset the influx of new elderly people. As a result of growth in per-capita costs, total costs will then begin to grow at an accelerating rate.

\section{Appendix 3.1}

Forecasting the disabled population is straightforward once we have constructed smooth age-prevalence profiles of disability. We cannot do so simply by averaging disability within single-year age categories. Even in large, nationally representative data sets, this results in very noisy profiles. To address this problem, we rely on the idea that disability prevalence should change smoothly across ages and years. Therefore, we take the raw age-specific estimates of disability and smooth them across ages and years using an overlapping polynomial method. ${ }^{6}$

Each observation $i$, taken in year ${ }_{i}$, consists of information about $i^{\prime}$ s selfreports regarding disability limitations $\delta_{i}$ and age $\left(a g e_{i}\right){ }^{7}$ As discussed earlier, an individual can be in one of three mutually exclusive disability states: institutionalized, no ADLs, or at least one ADL. Therefore, $\delta_{i}$ can take on one of three values, and so we need to construct age-prevalence profiles for all three levels of disability: $d(t, a ; 1), \ldots, d(t, a ; 3)$. To begin this process, we estimate the following multinomial logit model of disability using the combined NHIS/MCBS data from 1992 through 1996:

$$
\begin{aligned}
& \pi\left(\delta_{i}=1 a_{i}, t\right)=\frac{1}{1+\sum_{d=1}^{3} \exp \left(g_{1}\left(a_{i}\right) \beta_{1}^{d}+g_{2}(t) \beta_{2}^{d}\right)} \\
& \pi\left(\delta_{i}=2 a_{i}, \mathrm{t}\right)=\frac{\exp \left(g_{1}\left(a_{i}\right) \beta_{1}^{2}+g_{2}(t) \beta_{2}^{2}\right)}{1+\sum_{d=1}^{3} \exp \left(g_{1}\left(a_{i}\right) \beta_{1}^{d}+g_{2}(t) \beta_{2}^{d}\right)} \\
& \pi\left(\delta_{i}=3 a_{\mathrm{i}}, \mathrm{t}\right)=\frac{\exp \left(g_{1}\left(a_{i}\right) \beta_{1}^{3}+g_{2}(t) \beta_{2}^{3}\right)}{1+\sum_{d=1}^{3} \exp \left(g_{1}\left(a_{i}\right) \beta_{1}^{d}+g_{2}(t) \beta_{2}^{d}\right)}
\end{aligned}
$$


In effect, we calculate the prevalence of disability at each age and year in the context of a logistic distribution. The $g$ functions allow the presence of disability to vary with the year of observation and the agecohort of the respondent. Age and year enter the model through the functions, which are specified using an overlap polynomial.

The age polynomials are defined as:

$g_{1}\left(\right.$ age $\left._{i}\right)=\sum_{j=0}^{K}\left(\Phi\left(\frac{\text { age }_{i}-k_{j+1}}{\sigma_{1}}\right)-\Phi\left(\frac{\text { age }_{i}-k_{j}}{\sigma_{1}}\right)\right) p_{j}\left(\right.$ age $\left._{i} ; \beta_{1 j}\right)$.

where $p_{j}\left(\right.$ age $\left.e_{i} ; \beta_{1 j}\right) j=0, \ldots, K+1$ are all $n^{\text {th }}$-order polynomials in age $e_{i}{ }^{8}$ The terms $k_{0} \ldots k_{k+1}$ are called knots, and $\sigma_{1}$ is a smoothing parameter; all are fixed before estimation. With this smoothing technique, the knots define age intervals. When the smoothing parameter approaches zero, the age-profile over each interval simply equals the average disability level within that interval. In this case, the age-profile reduces to a step function, where each interval constitutes a separate step. ${ }^{9}$ As the smoothing parameter increases, the estimator uses increasingly more information from outside each interval. In the extreme, as the smoothing parameter approaches infinity, there is no meaningful distinction between any two intervals. Allowing nonzero values of the smoothing parameters eliminates the sharp discontinuity of the growth rates at the knots. One advantage of overlapping polynomials over traditional splines is that the function and all its derivatives are automatically continuous at the knots without imposing any parameter restrictions.

The overlapping polynomials for year, $g_{2^{\prime}}$ and its interaction with $g_{1}$ allow for flexible changes in the age-prevalence relationship over time. They are defined as:

$g_{2}\left(\right.$ year $\left._{i}\right)=\sum_{j=0}^{M}\left(\Phi\left(\frac{\text { year }_{i}-m_{j+1}}{\sigma_{2}}\right)-\Phi\left(\frac{\text { year }_{i}-m_{j}}{\sigma_{2}}\right)\right) q_{j}\left(\right.$ year $\left._{i} ; \beta_{2 j}\right)$

As before, the $m$ terms represent the knots, while the $\sigma$ term represents the smoothing parameter.

The object of the maximum likelihood logit estimation is to obtain consistent estimates for $\beta_{1}, \beta_{2}$ and $\beta_{3}-\hat{\beta}_{1}, \hat{\beta}_{2}$ and $\hat{\beta}_{3}$ respectively. Using these estimates, generating age-prevalence profiles representative for any particular year is a straightforward process. Let $d(t, a)$ be the disability prevalence among $a$-year-olds in year $t$. Then for $k=1,2,3$ :

$d(t, a ; k)=\frac{1}{N} \sum_{i} \Phi\left[d_{i}=k \mid\right.$ age $_{i}=a$, year $\left._{i}=t ; \hat{\beta}_{1}, \hat{\beta}_{2}, \hat{\beta}_{3}\right]$ 
We use this expression to construct smooth disability prevalence profiles for the years 1992 to 1996 . These are then used in turn as the basis for forecasting changes in disability according to the method discussed in the text.

\section{Notes}

This research was supported by the Health Care Financing Administration Centers for Medicare and Medicaid Services and the National Institute on Aging. The views expressed herein are solely those of the authors.

1. The presence of an activity limitation is identified by responses to another set of questions in which individuals are asked to name their major activity and then are asked if they are limited in their performance of that activity. Those who are limited are then asked the personal care question.

2. The sampling weights are adjusted accordingly.

3. For those age 65, we use the NHIS estimates rather than the MCBS estimates; 65-yearolds in the MCBS report artificially high rates of disability due to the sample design. The population under age 65 is disabled by definition because they are eligible for Medicare. Therefore, 65-year-olds include disabled 64-year-olds who age into the over-65 population. The sample is not refreshed quickly enough to eliminate this distortion.

4. We used the 1995 NHIS disability supplement to map the NHIS disability variables into ADL measures. Because no one in the NHIS is institutionalized, we then forecasted the prevalence of $1+$ ADLs in the community among the under-65 population.

5. See Manton and $\mathrm{Gu}$ (2001).

6. MaCurdy, Green, and Paarsch (1990) were the first to use this method in economics. Bhattacharya, Garber, and MaCurdy (1997) use this method to smooth cause-specific mortality profiles for the elderly.

7. The discussion assumes that $d_{i}$ refers to disability, but this method was also used for other diseases.

8. We use first-degree polynomials. Although we experimented with higher-order polynomials, we find that they add to the costs of computation with no change in the final results.

9. When this is the case, I (.) reduces to an indicator function equal to 0 if age $<k_{j}$ and 1 if age $\geq k_{j}$.

\section{References}

Bhattacharya, Jay, Alan Garber, and Thomas MaCurdy (1996). "Cause-Specific Mortality Among Medicare Enrollees," NBER working paper no. 5409.

Chernew, Michael, Richard Hirth, and David Cutler (2003). "Increased Spending on Health Care: How Much Can We Afford?" Health Affairs, 22(4):15-25. 
Goldman, Dana, Michael Hurd, Paul Shekelle, Sydne Newberry, Constantijn Panis, Baoping Shang, Jayanta Bhattacharya, Geoffrey Joyce, Darius Lakdawalla (2004). "Health Status and Medical Treatment of the Future Elderly: Final Report," RAND Working paper.

MaCurdy, Thomas, David Green, and Harry J. Paarsch (1990). "Assessing Empirical Approaches for Analyzing Taxes and Labor Supply," Journal of Human Resources, 25(3): $415-490$.

Manton, Kenneth G., Larry Corder, and Eric Stallard (1997). "Chronic Disability Trends in Elderly United States Populations: 1982-1994," Proceedings of the National Academy of Science, 94:2593-2598.

Manton, Kenneth G., and XiLiang Gu (2001). "Changes in the Prevalence of Chronic Disability in the United States Black and Nonblack Population Above Age 65 from 1982 to 1999," Proceedings of the National Academy of Science, published May 8. 